\title{
Haptoglobin Decreased, CTCAE
}

National Cancer Institute

\section{Source}

National Cancer Institute. Haptoglobin Decreased, CT CAE. NCI Thesaurus. Code C143526.

A finding based on laboratory test results that indicate an decrease in levels of haptoglobin in a blood specimen. 